\title{
Image Registration Using SIFT and Canonical Correlation Analysis
}

\author{
Wei Zhao ${ }^{1, \text { a }}$, Zheng Tian ${ }^{1,2, b}$, Lijuan Yang ${ }^{1, \mathrm{c}}$, Weidong Yan ${ }^{1, \mathrm{~d}}$ and Jinhuan \\ Wen $^{1, e}$ \\ ${ }^{1}$ Departments of Applied Mathematics, Northwestern Polytechnical University, Xi'an, 710129, China \\ ${ }^{2}$ State Key Laboratory of Remote Sensing Science, Institute of Remote Sensing and Digital Earth, \\ CAS, Beijing, 100101, China \\ awu.kong1@163.com, bzhtian@nwpu.edu.cn, cyanglijuan1987@163.com, dyanweidong@nwpu.edu \\ .cn, êwjhgdsx@nwpu.edu.cn
}

Keywords: Image registration, Feature matching, SIFT, Mismatches removal, RANSAC

\begin{abstract}
To improve the quality of SIFT feature matching, a mismatches removal method is proposed based on the collinearity property of the canonical correlation features. The influence of each match on the collineartiy degree is analyzed to find false matches. Firstly, a putative set of matches is obtained based on distances between SIFT feature descriptors. Secondly, the spatial relationship of matched points is encoded by canonical correlation analysis. The match with the largest effect on the collinearity degree is iteratively eliminated. Experimental results show the proposed method can reserve a high rate of correct matches determined by a threshold. Compared with several other mismatches removal methods, it provides comparable or better results.
\end{abstract}

\section{Introduction}

For the past few decades, image registration is involved widely many applications including image mosaic, change detection, cartography and so on [1]. In feature based image registration methods, feature detection and feature matching are two key steps [2]. Some sophisticated feature detection methods have been applied successfully in image registration [3, 4]. However, since there are usually areas with similar intensity distribution in the reference and sensed images, these local descriptor based matching process will result in mismatches inevitably.

To address the issue, Euclidean distance ratio filter [3] is often used to remove unreliable matches in SIFT matching by thresholding the Euclidean distance ratio of closest to second-closest neighbors. However, there are many mismatches if the threshold is high and many correct matches are excluded if the threshold is low. So a convenient mode-seeking algorithm which exploits the scale, orientation, and position information of SIFT features was presented in [5]. More recently, Yan et al. [6] fit a line using the first pair of (kernel) CCA features and remove mismatches by thresholding the distances from points to the line. We will refer the method as CCA-I. The main drawback of these methods is that they tend to obtain higher accuracy at the cost of reserving fewer matches. This leads to the removal of high quality matches and suppresses the registration performance. To reserve more matches, although Random Sample Consensus (RANSAC) [7] is a classic and effective method in image registration [8], its randomness a thorny problem. To reduce the randomness, an Optimized Random Sampling Algorithm (ORSA) was proposed for SIFT based image registration [9].

In this paper, we proposed a mismatches removal method following CCA-I [6] with the aim of detecting as many mismatches as possible while keeping as many correct matches as possible. The method improves CCA-I in two aspects. Firstly, since only the first pair of CCA features is usually not enough to indicate all the mismatches, more CCA features are considered. Secondly, because simple thresholding cannot find all mismatches and the performance also depends on the line fitting scheme in CCA-I, a different but effective collinearity criterion is used to overcome the drawback. 


\section{A Review of Canonical Correlation Analysis}

Canonical correlation analysis (CCA) has been a successful method for data analysis in computer vision problems [10]. Given the reference and sensed images, let $X=\left\{x_{1}, x_{2}, \cdots, x_{n}\right\}$ and $Y=\left\{y_{1}, y_{2}, \cdots, y_{n}\right\}$ be the position vectors of one-to-one correspondence pairs obtained from SIFT. Their mean vectors are $\mu_{x}$ and $\mu_{y}$. Their within-set covariance matrices are $C_{x}$ and $C_{y}$. The between-set covariance matrix is denoted by $C_{x y}$. The aim of CCA is to find two directions $u$ and $v$, such that the correlation of the two projections $s_{i}=u^{T}\left(x_{i}-\mu_{x}\right), 1 \leq i \leq n$ and $t_{i}=v^{T}\left(y_{i}-\mu_{y}\right), 1 \leq i \leq n$, is maximized. $u$ and $v$ can be obtained from the eigenvalue problems:

$$
\begin{aligned}
& C_{x}^{-1} C_{x y} C_{y}^{-1} C_{x y}^{T} u=r^{2} u, \\
& v=(1 / r) C_{y}^{-1} C_{x y}^{T} u .
\end{aligned}
$$

Finally, they are normalized by the constraints $u^{T} C_{x} u=v^{T} C_{y} v=1 . r$ is the canonical correlation coefficient. The projections of $X$ and $Y$ on the first pair of eigenvectors $u$ and $v$ are referred as the first pair of canonical correlation features. After that, the second pair of canonical correlation features are obtained from the second pair of eigenvectors. The canonical correlation features are invariant with affine transformations which are commonly used transformation model in image registration.

\section{The Proposed Method}

Because $\left(s_{i}, t_{i}\right), i=1,2, \cdots, n$ are collinear when the original point sets are linearly dependent, CCA-I [6] used the linear relationship of the first pair of (kernel) CCA features to remove mismatches. Given the paired points $\left(s_{i}, t_{i}\right), i=1,2, \cdots, n$ obtained from (kernel) CCA, a line is fit and the distances from points to the line are thresholded to indicate mismatches. However, the method depends much on the performance of line fitting and the threshold. So a method that iteratively eliminates mismatches is obtained by analyzing the impact of each match on the collinearity degree.

Given the points $\left(s_{i}, t_{i}\right), i=1,2, \cdots, n$, if the eigenvalues of their covariance matrix $C_{s t}$ in descending order are $\lambda_{1}$ and $\lambda_{2}$, respectively, the collinearity degree is defined as $\tau=1-\lambda_{2} / \lambda_{1}$, where the geometric meanings of $\lambda_{1}$ and $\lambda_{2}$ are the mean square lengths of the projections on the first and second principal components of $\left(s_{i}, t_{i}\right), i=1,2, \cdots, n$.

Analysis of the Effect on the Collinearity Degree by Removing a Match. Let $\bar{X}_{i}=X \backslash\left\{x_{i}\right\}$ and $\bar{Y}_{i}=Y \backslash\left\{y_{i}\right\}$ be the point sets after removing the ith match $\left(x_{i}, y_{i}\right)$. Then their between-set covariance matrix $C_{x y}^{i}$ is derived from $C_{x y}$ by some algebraic computations:

$$
C_{x y}^{i}=\frac{n}{n-1} C_{x y}-\frac{n}{(n-1)^{2}}\left(x_{i}-\mu_{x}\right)\left(y_{i}-\mu_{y}\right)^{T} .
$$

A similar recursion formula holds for the within-set covariance matrices $C_{x}^{i}$ and $C_{y}^{i}$ by replacing $x$ with $y$ or $y$ with $x$ in Eq. 2 .

Substitute $C_{x}^{i}, C_{y}^{i}$ and $C_{x y}^{i}$ into Eq. 1 to compute the changed projection directions $u$ and $v$ after removing the $i$ th match. It is unnecessary to recompute $\left(s_{j}, t_{j}\right), j=1, \cdots, i-1, i+1, \cdots, n$ for the current collinearity degree because the quadratic characteristic polynomial of $C_{s t}^{i}$ can be computed directly 
as $\lambda^{2}-2 \lambda+\left(1-r^{2}\right)$ after some algebraic computations. One can obtain the eigenvalues of $C_{s t}^{i}$ and they are $\lambda_{1}=1+r$ and $\lambda_{2}=1-r$. So the current collinearity degree is $\tau_{i}=2 r /(1+r)$. The average of the collinearity degrees corresponding to the first and second canonical correlation coefficients is used in this paper, that is $\tau_{i}=r_{1} /\left(1+r_{1}\right)+r_{2} /\left(1+r_{2}\right)$.

The collinearity degrees $\tau_{i}, i=1,2, \cdots, n$ are computed for all the matches $\left(x_{i}, y_{i}\right), i=1,2, \cdots, n$ and the index $i_{0}$ corresponding to the largest collinearity degree is deemed as a mismatch. It is canceled, and $C_{x}, C_{y}$ and $C_{x y}$ are updated by $C_{x}^{i_{0}}, C_{y}^{i_{0}}$ and $C_{x y}^{i_{0}}$, respectively. $\mu_{x}$ and $\mu_{y}$ are also updated for the next iteration using the relation $n \mu_{z}^{\text {old }}-(n-1) \mu_{z}^{\text {new }}=z_{i_{0}}$, where $z=x$ or $y$.

The above process is repeated to find the next mismatch until the collinearity degree of the remaining matches exceeds a certain threshold $T$. Higher threshold $T$ leads to lower RMSE but fewer matches. However, the method will keep matches satisfying the threshold as more as possible.

\section{Experimental Results}

In this section, we compare the proposed method with CCA-I [6], RANSAC [7], ORSA [9] and the mode-seeking method [5] on three different image pairs with more mismatches. The threshold for SIFT initial matching is 0.8 in these experiments.

Fig. 1 (a)-(c) and (g)-(i) illustrate the matching processs of two pairs of Landsat TM images, and (d)-(f) a pair of optical images. Using the proposed method, the false matches can be iteratively eliminated, and the removal orders are shown in Fig. 1(b), (e) and (h). The range of the corresponding collinearity degree is normalized to $[0,1]$. The mismatches found are labeled in Fig. 1(a), (d) and (g). Root mean square errors (RMSE) and the number of matches are summarized in Table 1 . It can be seen that the proposed method performs better than CCA-I, RANSAC and ORSA in both matching quantity and quality. Especially, it has great improvement compared with CCA-I. Though the RMSE for Fig. 1(d) is slightly greater than the mode-seeking method, the matches found are more. In fact, if we set a higher threshold $T=1-4 \times 10^{-5}$ for Fig. $1(\mathrm{~d})$, the result becomes $196 / 1.6521$, which is better than the mode-seeking method. 


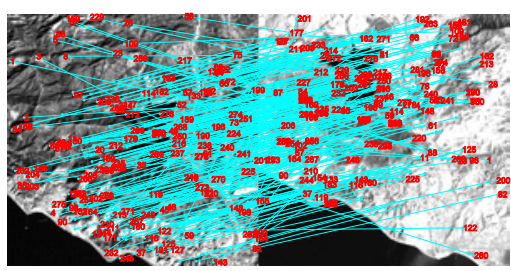

(a)

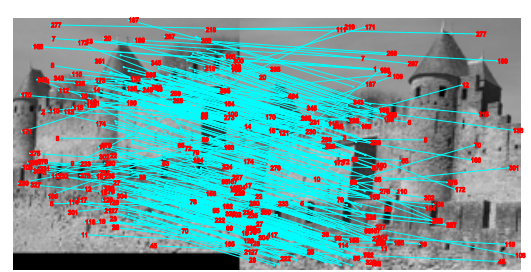

(d)

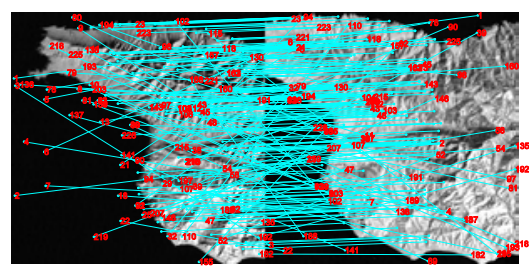

(g)

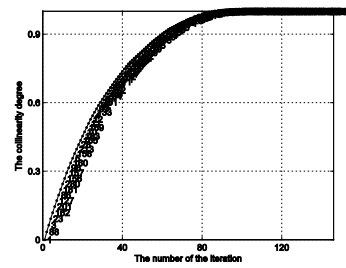

(b)

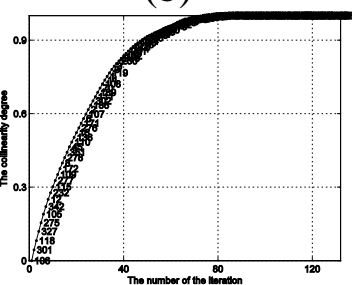

(e)

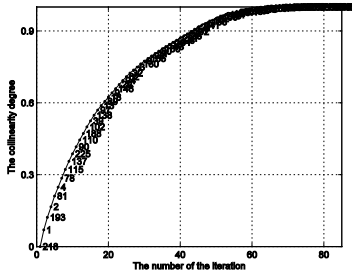

(h)

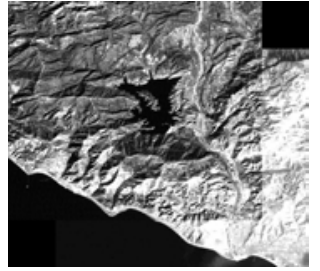

(c)

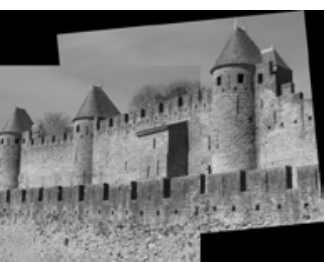

(f)

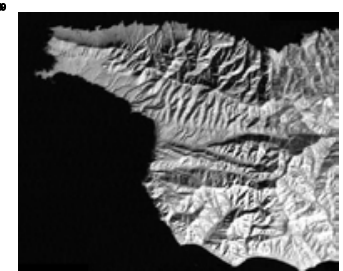

(i)

Fig. 1 The matching process of the proposed method. (a, d, g) Matches found using SIFT. (b, e, h) The collineartiy degrees of the CCA features corresponding to the removal order. (c, f, i) The registration result using the final matches.

Table 1 Performance comparison of the five mismatches removal methods

\begin{tabular}{|c|c|c|c|}
\hline \multirow{2}{*}{ Methods } & \multicolumn{3}{|c|}{ Number of Matches/RMSE } \\
\cline { 2 - 4 } & Fig. 1(a) & Fig. 1(d) & Fig. 1(g) \\
\hline The proposed method & $135 / 1.0031$ & $221 / 1.8973$ & $141 / 1.6056$ \\
\hline CCA-I & $119 / 5.0469$ & $160 / 6.7341$ & $52 / 3.8389$ \\
\hline RANSAC & $126 / 1.1311$ & $207 / 2.2039$ & $132 / 1.6417$ \\
\hline ORSA & $121 / 1.0569$ & $215 / 2.3811$ & $125 / 1.6337$ \\
\hline Mode-Seeking & $63 / 1.7462$ & $193 / 1.7822$ & $141 / 1.7845$ \\
\hline
\end{tabular}

\section{Conclusion}

In this paper, a mismatches removal algorithm, inspired by the collinearity property of the CCA features, is proposed to improve the accuracy of the SIFT-based method for image registration. The method is deterministic and applicable to various image registration problems. It iteratively eliminates match which has the largest influence on the collinearity degree in order to obtain consensus matching. The experimental results on different image pairs demonstrate the method's advantage that it reserved more correct matches with low RMSE.

\section{Acknowledgments}

This work was supported by the Natural Science Foundation of China (No.60972150, No.61201323 and No.61301196), Natural Science Foundation projects of Shaanxi Province (No. 2014JQ5189), and the Open Fund of State Key Laboratory for Remote Sensing Science (No. OFSLRSS201206). 


\section{References}

[1] J. Le Moigne, N. S. Netanyahu, and R. D. Eastman, Image Registration for Remote Sensing, Cambridge University Press, 2011.

[2] A. Goshtasby, 2-D and 3-D Image Registration for Medical, Remote Sensing, and Industrial Applications, Wiley-Interscience, New York, 2005.

[3] D. G. Lowe, Distinctive image features from scale-invariant keypoints, Int. J. Comput. Vision. 60(2004) 91-110.

[4] L. Yang, Z. Tian, and W. Zhao, A new affine invariant feature extraction method for SAR image registration, Int. J. Remote. Sens. 35(2013) 7219-7229.

[5] B. Kupfer, N. S. Netanyahu, and I. Shimshoni, An efficient SIFT-based mode-seeking algorithm for sub-pixel registration of remotely sensed images, IEEE Geosci. Remote. S. 12(2015) 379-383.

[6] W. Yan, H. She, and Z. Yuan, Robust registration of remote sensing image based on SURF and KCCA, J. Indian. Soc. Remote. 42(2014) 291-299.

[7] M. A. Fischler and R. C. Bolles, Random sample consensus: a paradigm for model fitting with applications to image analysis and automated cartography, Commun. Acm. 24(1981) 381-395.

[8] T. Kim and Y. J. Im, Automatic satellite image registration by combination of matching and random sample consensus. IEEE T. Geosci. Remote. 41(2003) 1111-1117.

[9] L. Moisan, P. Moulon, and P. Monasse, Automatic homographic registration of a pair of images, with a contrario elimination of outliers, Image Processing On Line, 2(2012) 56-73.

[10] R. Lan, J. Yang, Y. Jiang, Z. Song, and Y. Tang, An affine invariant discriminate analysis with canonical correlation analysis, Neurocomputing, 86(2012) 184-192. 